УДК 621.373.826.032:534.232.082.73

[0000-0001-7529-9076] О. М. Петрищев, д.т.н., професор,

Державне підприємство «Київський державний науково-дослідний інститут гідроприладів» ${ }^{[0000-0002-1571-401 X]}$ К. В. Базіло, к.т.н., доиент

Черкаський державний технологічний університет б-р Шевченка, 460, Черкаси, 18006, Україна

\title{
ПОБУДОВА МАТЕМАТИЧНОЇ МОДЕЛІ БІМОРФНОГО П'ЄЗОЕЛЕКТРИЧНОГО ТРАНСФОРМАТОРА
}

Рімення задачі про збудження коливань поперечного вигину в біморфному п'єзоелектричному трансформаторі наразі є актуальним для багатьох галузей, де застосовуються пристрої на основі n'єзоматеріалів. Кіниевою метою математичного моделювання фізичного стану коливальних п'єзокерамічних елементів є якісний $і$ кількісний опис характеристик $і$ параметрів існуючих у них електричних $і$ пружних полів. Математична модель п'єзоелектричних трансформаторів, що прачюють з використанням коливань поперечного вигину в біморфному п'єзоелектричному елементі, побудована з мінімальною кількістю припущень, які спрощують реальну ситуачію. Це дає можливість стверджувати, щз запропонована схема побудови доставляє математичні моделі, достатньою мірою адекватні реальним об'єктам і існуючим у них фізичним процесам. Розглянуто конструкиію і особливості математичного опису біморфного п'єзоелектричного елемента, принцип дії якого базується на використанні вісесиметричних коливань поперечного вигину; отримано рішення задачі про порушення коливань поперечного вигину в біморфному п'єзоелектричному елементі. Побудовано математичну модель дискового біморфного п'єзоелектричного трансформатора з симетричним електричним навантаженням у вторинному електричному колі з урахуванням повного набору геометричних, фізико-механічних та електричних параметрів. В результаті дослідження математичної моделі реального пристрою можна визначити той набір геометричних, фізико-механічних та електричних параметрів реального об'єкта, який забезпечує реалізацію технічних показників функиіонального елемента п'єзоелектроніки, обумовлених у технічному завданні. Вартість збережених ресурсів становить комериійну ціну математичної моделі.

Ключові слова: n'єзоелектричний трансформатор, біморфний елемент, фізичні процеси, математичний опис.

Вступ. П'єзокераміка завдяки своїм унікальним властивостям має застосування в різних галузях техніки і технології. Виробники п'єзокераміки, елементів і компонентів на ii основі, намагаючись повніше задовольнити сучасні вимоги ринку, проводять дослідження і конструкторські роботи з метою поліпшення параметрів кераміки, розробляють іiї нові типи, на що виділяються значні фінансові кошти. 3 метою здешевлення продукції розробляються нові технології, що є більш енергозберігаючими і дають змогу автоматизувати процеси виробництва $[1,2]$.

У силовій електроніці електромагнітні трансформатори були домінуючим компонентом для перетворення електроенергії. Тенденція силових перетворювачів йде в напрямі вищої ефективності і меншого об'єму. Дослідження показали, що п'єзоелектричні транс-

форматори (ПТ) можуть конкурувати з традиційними електромагнітними трансформаторами як за ефективністю, так і за густиною потужності [3-6]. Тому ПТ є перспективною галуззю досліджень [7].

П'єзоелектричні трансформатори широко застосовуються в радіоелектроніці. Відсутність у ПТ обмоток і магнітної системи, можливість застосування планарної конструкції, висока економічність і надійність істотно поліпшують масогабаритні показники радіоелектронних вузлів $[8,9]$. Найбільше застосування ПТ знаходять у високовольтних джерелах вторинного електроживлення (ДВЕЖ) і в пристроях, де потрібен великий коефіцієнт перетворення напруги, зокрема в медичній техніці [10].

Метою роботи $\epsilon$ рішення задачі про збудження коливань поперечного вигину в біморфному п'єзоелектричному трансформаторі. 
Огляд літератури. Існує безліч публікацій, присвячених створенню та дослідженню математичних моделей п'єзоелектричних перетворювачів. Починаючи 3 монографії [11], основи розрахунку передавальних характеристик п'єзоелектричних перетворювачів розглядалися, наприклад, в роботах [12-15].

Однак у багатьох роботах описані тільки процеси, що відбуваються в п'єзоелектричному диску з поверхнею, яка повністю покрита електродами. Існує також ряд робіт різнорідного характеру, присвячених рішенню задачі електромеханічних коливань п'єзоелементів 3 поділеними електродами (трансформаторного типу).

Роботи, присвячені розрахунку параметрів і характеристик п'єзоелектричних перетворювачів, можна розділити на дві групи. Перша група включає роботи, що грунтуються на польовій (як у роботі [16]) моделі п'єзокерамічного диска, наприклад [12, 13]. Друга група включає роботи, що базуються на використанні еквівалентних схем, наприклад [14, 15]. Математичні моделі п'єзоелектричних перетворювачів, побудовані на основі аналізу так званих еквівалентних кіл, не враховують той очевидний факт, що рухи матеріальних частинок п'єзоелектричного диска повинні задовольняти другому i третьому законам Ньютона. Ігнорування цієї фундаментальної позиції виводить ці моделі за межі механіки деформованого твердого тіла i, як наслідок, гарантовано визначає їх неадекватність реальним об'єктам і фізичним процесам, що відбуваються в них.

Тому актуальним завданням $є$ розробка математичної моделі біморфного п'єзоелектричного трансформатора.

Матеріали та методи. Розглянемо конструкцію дискового п'єзоелектричного трансформатора, розрахункову схему якого зображено на рисунку 1.

На рисунку 1 позицією 1 показана нерухома опора, позицією 2 - металевий диск, позицією 3 - два однакові п'єзокерамічні диски. Поверхні дисків, які знаходяться в механічному контакті $з$ металевим диском, повністю електродовані і приклеєні високомолекулярним струмопровідним клеєм до металевої круглої пластини. Металева пластина заземлена. На зовнішніх поверхнях п'єзокерамічних дисків розташовуються два кільцеві електроди. На рисунку 1, $б$ зображено вигляд зверху на дисковий біморфний п'єзоелектричний трансформатор. Позицією 4 на рисунку 1, 6 показаний кільцевий електрод первинного електричного кола. Точно такий же електрод $\epsilon$ на зовнішній поверхні нижнього п'єзокерамічного диска. На цю пару кільцевих електродів подається різниця електричних потенціалів від джерела електричних сигналів з вихідним електричним опором $Z_{1}$.

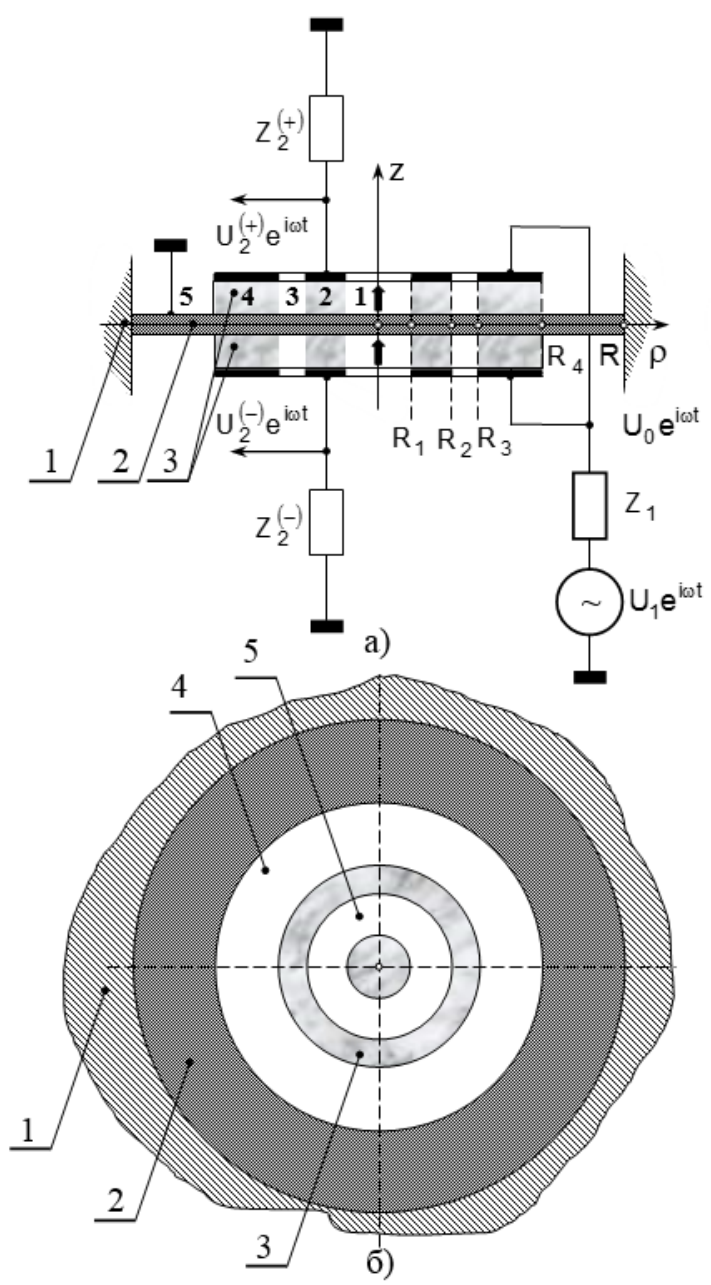

Рисунок 1 - Розрахункова схема біморфного дискового п'єзоелектричного трансформатора

Напрямки електричної поляризації п'єзокерамічних дисків зображено на рисунку 1 , $a$ жирними стрілками. При такому механічному поєднанні п'єзокерамічних дисків електричний потенціал $U_{0} e^{\text {iøt }}$ на парі кільцевих електродів первинного електричного кола завдяки зворотному п'єзоелектричному ефекту створює згинальні моменти в області № 4, які змінюються в часі за законом $e^{\text {iөt }}$ (рисунок $1, a$ ), тобто в кільці $R_{3} \leq \rho \leq R_{4}$. Під дією цих згинальних моментів вся конструкція починає здійснювати осесиметричні коливання поперечного вигину. Виникаючі при 
цьому деформації формують в об'ємі п'єзокерамічних дисків змінну в часі за законом $e^{\text {iøt }}$ електричну поляризацію. На кільцевих електродах вторинного електричного кола дискового п'єзоелектричного трансформатора (позиція 5 на рисунку 1, б) виникають електричні заряди, які своїм електричним полем змушують рухатися вільні носії електрики в провідниках, які з'єднують електричні навантаження (символи $Z_{2}^{( \pm)}$на рисунку $1, a$ ) з електродами вторинного електричного кола. В результаті проходження електричного струму на електричних навантаженнях $Z_{2}^{( \pm)}$формуються електричні потенціали $U_{2}^{( \pm)} e^{\text {iøt }}$, які $\epsilon$ вихідними електричними сигналами розглянутого функціонального елемента п'єзоелектроніки.

У загальному випадку можлива ситуація, коли $Z_{2}^{(+)} \neq Z_{2}^{(-)}$. Внаслідок цього амплітудні значення вихідних потенціалів $U_{2}^{(+)} \neq U_{2}^{(-)}$. Це змушує визначити математичну модель дискового біморфного п'єзоелектричного трансформатора в такий спосіб:

$$
K^{( \pm)}=\frac{U_{2}^{( \pm)}}{U_{1}} .
$$

Вже неодноразово було показано, що обчислення коефіцієнта трансформації, тобто побудова математичної моделі п'єзоелектричного трансформатора, неминуче стикається 3 проблемою визначення коефіцієнта електроакустичного зв'язку між потенціалами на електродах вторинного та первинного електричного кола. Ця проблема ефективно вирішується після визначення в явному вигляді співвідношень для розрахунку компонентів вектора зміщення матеріальних частинок п'єзоелектрика в різних областях (вільних від електродів і з електродами на поверхні) п'єзоелектричного трансформатора.

Визначимо зміщення матеріальних частинок у різних областях п'єзокерамічних дисків трансформатора.

На рисунку 1, а цифрами 1, 2, 3 і 4 пронумеровані різні за своїми фізичними властивостями області п'єзокерамічних дисків.

В областях непарних номерів відсутне електродне покриття на зовнішніх поверхнях дисків. 3 цієї причини в об’ємі областей № 1 i № 3 аксіальний компонент $D_{z}^{( \pm)}$вектора електричної індукції дорівнюе нулю, і фізичний стан цих областей повністю визначається уза- гальненим законом Гука, який у разі тонких дисків, що коливаються в вакуумі, записується в наступному вигляді:

$$
\begin{array}{r}
{ }^{(n)} \sigma_{\rho \rho}^{( \pm)}=-z c_{11}^{D}\left(\frac{\partial^{2} w_{n}}{\partial \rho^{2}}+\frac{k_{D}}{\rho} \frac{\partial w_{n}}{\partial \rho}\right), \quad n=1 ; 3, \\
{ }^{(n)} \sigma_{\phi \phi}^{( \pm)}=-z c_{11}^{D}\left(k_{D} \frac{\partial^{2} w_{n}}{\partial \rho^{2}}+\frac{1}{\rho} \frac{\partial w_{n}}{\partial \rho}\right),{ }^{(n)} D_{z}^{( \pm)}=0,
\end{array}
$$

де $w_{n}$ - прогин в області номера $n$; $c_{11}^{D}-$ модуль пружності .

В областях 3 парними номерами $m=2 ; 4$ фізичний стан деформованої п'єзокераміки визначається наступними співвідношеннями:

$$
\begin{aligned}
& { }^{(m)} \sigma_{\rho \rho}^{( \pm)}=-z c_{11}^{D}\left(\frac{\partial^{2} w_{m}}{\partial \rho^{2}}+\frac{k_{D}}{\rho} \frac{\partial w_{m}}{\partial \rho}\right) \pm \\
& \pm(h+\alpha / 2) \frac{\left(e_{31}^{*}\right)^{2}}{\chi_{33}^{\sigma}} \frac{1}{\rho} \frac{\partial}{\partial \rho}\left(\rho \frac{\partial w_{m}}{\partial \rho}\right) \pm e_{31}^{*} \frac{U_{m}^{( \pm)}}{\alpha} \\
& { }^{(m)} \sigma_{\phi \phi}^{( \pm)}=-z c_{11}^{D}\left(k_{D} \frac{\partial^{2} w_{m}}{\partial \rho^{2}}+\frac{1}{\rho} \frac{\partial w_{m}}{\partial \rho}\right) \pm \\
& \pm(h+\alpha / 2) \frac{\left(e_{31}^{*}\right)^{2}}{\chi_{33}^{\sigma}} \frac{1}{\rho} \frac{\partial}{\partial \rho}\left(\rho \frac{\partial w_{m}}{\partial \rho}\right) \pm e_{31}^{*} \frac{U_{m}^{( \pm)}}{\alpha}, \\
& { }^{(m)} D_{z}^{( \pm)}=\mp(h+\alpha / 2) \frac{\left(e_{31}^{*}\right)^{2}}{\chi_{33}^{\sigma}} \frac{1}{\rho} \frac{\partial}{\partial \rho}\left(\rho \frac{\partial w_{m}}{\partial \rho}\right) \mp \chi_{33}^{\sigma} \frac{U_{m}^{( \pm)}}{\alpha}, \\
& m=2 ; 4,
\end{aligned}
$$

де $U_{4}^{( \pm)}=U_{0}$.

У металевій пластині напруженодеформований стан визначається стандартним чином:

$$
\begin{gathered}
{ }^{(k)} \sigma_{\rho \rho}^{( \pm)}=-\frac{E z}{1-v^{2}}\left(\frac{\partial^{2} w_{k}}{\partial \rho^{2}}+\frac{v}{\rho} \frac{\partial w_{k}}{\partial \rho}\right), k=1,2,3,4,5, \\
{ }^{(k)} \sigma_{\phi \phi}^{( \pm)}=-\frac{E z}{1-v^{2}}\left(v \frac{\partial^{2} w_{k}}{\partial \rho^{2}}+\frac{1}{\rho} \frac{\partial w_{k}}{\partial \rho}\right),
\end{gathered}
$$

де область № 5 - це кільце $R_{4} \leq R$ (рисунок $1, a$ ).

Лінійна густина згинальних моментів $M_{\rho}^{(k)}(k=1, \ldots, 5)$ задається наступними співвідношеннями:

- області п'єзокерамічних дисків 3 непарними номерами $n=1 ; 3$ :

$$
M_{\rho}^{(n)}=-D_{1}^{(n)}\left(\frac{\partial^{2} w_{n}}{\partial \rho^{2}}+\frac{\eta_{n}}{\rho} \frac{\partial w_{n}}{\partial \rho}\right),
$$

де згинальна жорсткість

$$
D_{1}^{(n)}=\frac{2}{3} c_{11}^{D}\left[(\alpha+h)^{3}-h^{3}\right]+\frac{2 E h^{3}}{3\left(1-v^{2}\right)} ; \eta_{n}=\frac{D_{2}^{(n)}}{D_{1}^{(n)}} ;
$$




$$
D_{2}^{(n)}=\frac{2}{3} c_{12}^{D}\left[(\alpha+h)^{3}-h^{3}\right]+\frac{2 E v h^{3}}{3\left(1-v^{2}\right)} ;
$$

- області дисків 3 парними номерами $m=2 ; 4$ :

$$
M_{\rho}^{(m)}=-D_{1}^{(m)}\left(\frac{\partial^{2} w_{m}}{\partial \rho^{2}}+\frac{\eta_{m}}{\rho} \frac{\partial w_{m}}{\partial \rho}\right)+M_{0}^{(m)},
$$

де $D_{1}^{(m)}=D_{n э}-D_{0}+D_{\mu} ; \eta_{m}=D_{2}^{(m)} / D_{1}^{(m)}$;

$D_{2}^{(m)}=k_{d} D_{n э}-D_{0}+v D_{M} ; D_{n э}=\frac{2}{3} c_{11}^{D}\left[(\alpha+h)^{3}-h^{3}\right]$;

$D_{0}=2 \alpha(h+\alpha / 2)^{2} \frac{\left(e_{31}^{*}\right)^{2}}{\chi_{33}^{\sigma}} ; D_{M}=\frac{2 E h^{3}}{3\left(1-v^{2}\right)} ; M_{0}^{(m)}-$

додаткові згинальні моменти, обумовлені зворотним п'єзоелектричним ефектом. При цьому $M_{0}^{(2)}=e_{31}^{*}(h+\alpha / 2)\left[U_{2}^{(-)}+U_{2}^{(+)}\right] \mathrm{i}$ $M_{0}^{(4)}=2 e_{31}^{*}(h+\alpha / 2) U_{0}$.

Лінійна густина згинального моменту в межах металевого кільця $R_{4} \leq \rho \leq R$ визначається стандартним чином:

$$
M_{\rho}^{(5)}=-D_{M}\left(\frac{\partial^{2} w_{5}}{\partial \rho^{2}}+\frac{v}{\rho} \frac{\partial w_{5}}{\partial \rho}\right) .
$$

Лінійна густина поперечних сил $Q_{k}$ на $k$-ій ділянці біморфного п'єзоелектричного трансформатора розраховується за формулою

$Q_{k}=D_{1}^{(k)}\left(\frac{\partial^{3} w_{k}}{\partial \rho^{3}}+\frac{1}{\rho} \frac{\partial^{2} w_{k}}{\partial \rho^{2}}-\frac{1}{\rho^{2}} \frac{\partial w_{k}}{\partial \rho}\right), k=1, \ldots, 5$,

де $D_{1}^{(5)} \equiv D_{\mu}$.

Загальне рішення рівняння сталих гармонійних коливань поперечного вигину для різних областей біморфного дискового п'єзоелектричного трансформатора має наступний вигляд:

$$
\begin{gathered}
w_{1}(\rho)=A_{1} J_{0}\left(\lambda_{1} \rho\right)+A_{2} I_{0}\left(\lambda_{1} \rho\right), \\
w_{2}(\rho)=A_{3} J_{0}\left(\lambda_{2} \rho\right)+A_{4} N_{0}\left(\lambda_{2} \rho\right)+A_{5} I_{0}\left(\lambda_{2} \rho\right)+A_{6} K_{0}\left(\lambda_{2} \rho\right), \\
w_{3}(\rho)=A_{7} J_{0}\left(\lambda_{1} \rho\right)+A_{8} N_{0}\left(\lambda_{1} \rho\right)+A_{9} I_{0}\left(\lambda_{1} \rho\right)+A_{10} K_{0}\left(\lambda_{1} \rho\right), \\
w_{4}(\rho)=A_{11} J_{0}\left(\lambda_{2} \rho\right)+A_{12} N_{0}\left(\lambda_{2} \rho\right)+A_{13} I_{0}\left(\lambda_{2} \rho\right)+A_{14} K_{0}\left(\lambda_{2} \rho\right), \\
w_{5}(\rho)=A_{15} J_{0}(\lambda \rho)+A_{16} N_{0}(\lambda \rho)+A_{17} I_{0}(\lambda \rho)+A_{18} K_{0}(\lambda \rho),(10)
\end{gathered}
$$$$
\text { де } A_{1}, \ldots, A_{18} \text { - константи, що підлягають ви- }
$$
значенню; $\lambda_{1}$ i $\lambda_{2}-$ хвильові числа згинальних коливань у непарних і парних за номером областях активної зони біморфного п'єзоелектричного елемента; $\lambda$ - хвильове число згинальних коливань металевого кільця $R_{4} \leq \rho \leq R$. Хвильові числа задаються формулами: $\lambda_{1}=\sqrt[4]{2\left(h \rho_{M}+\alpha \rho_{n \ni}\right) \omega^{2} / D_{1}^{(1)}}$, $\lambda_{2}=\sqrt[4]{2\left(h \rho_{M}+\alpha \rho_{n э}\right) \omega^{2} / D_{1}^{(2)}}, \quad \lambda=\sqrt[4]{2 h \rho_{\mu} \omega^{2} / D_{\mu}}$, де $\rho_{\mu}$ i $\rho_{n э}$ - густина металевої пластинки i п'єзокераміки.

Коефіцієнти $A_{1}, \ldots, A_{18}$, що входять до складу загальних рішень (10), повинні забезпечувати безперервність (зшивання) характеристик напружено-деформованого стану різних областей п'єзоелектричного трансформатора на умовних межах $\rho=R_{1}, \ldots, R_{4}$. Цей факт записується в такий спосіб:

$$
\begin{gathered}
w_{j}\left(R_{j}\right)=w_{j+1}\left(R_{j}\right), \\
\left.\frac{\partial w_{j}(\rho)}{\partial \rho}\right|_{\rho=R_{j}}=\left.\frac{\partial w_{j+1}(\rho)}{\partial \rho}\right|_{\rho=R_{j}}, \\
M_{\rho}^{(j)}\left(R_{j}\right)=M_{\rho}^{(j+1)}\left(R_{j}\right), \\
Q_{j}\left(R_{j}\right)=Q_{j+1}\left(R_{j}\right), \quad j=1,2,3,4 .
\end{gathered}
$$

Умови (11) доставляють 16 алгебраїчних рівнянь, до складу яких входять 18 коефіцієнтів $A_{1}, \ldots, A_{18}$, що підлягають визначенню. Відсутні два рівняння доставляють умови закріплення контуру $\rho=R$ металевої пластинки. Будемо вважати, що контур $\rho=R$ жорстко защемлений. Це означає, що повинні бути виконані такі умови:

$$
\begin{gathered}
w_{5}(R)=0, \\
\left.\frac{\partial w_{5}(\rho)}{\partial \rho}\right|_{\rho=R}=0 .
\end{gathered}
$$

Умови зшивання рішень (11) і граничні умови (12) утворюють неоднорідну систему 3 18 лінійних алгебраїчних рівнянь, до складу якої входить рівно 18 шуканих констант $A_{1}, \ldots, A_{18}$. Ця система рівнянь вирішується відносно шуканих констант єдиним чином. Символічний запис цієї системи рівнянь має наступний вигляд:

$$
m_{j k} A_{k}=P_{j}, \quad j, k=1, \ldots, 18 .
$$

При запису системи рівнянь (13) передбачається виконання підсумовування по двічі повторюваному індексу $k$. Коефіцієнти $m_{j k}$ i праві частини $P_{j}$ в системі рівнянь (13) визначаються наступними виразами:

$$
\begin{gathered}
m_{11}=J_{0}\left(\Omega_{11}\right) ; \Omega_{11}=\lambda_{1} R_{1} ; m_{12}=I_{0}\left(\Omega_{11}\right) ; \\
m_{13}=-J_{0}\left(\Omega_{21}\right) ; \Omega_{21}=\lambda_{2} R_{1} ; \\
m_{14}=-N_{0}\left(\Omega_{21}\right) ; m_{15}=-I_{0}\left(\Omega_{21}\right) ; m_{16}=-K_{0}\left(\Omega_{21}\right) ; \\
m_{17} \div m_{1,18}=0 ; P_{1}=0 ; m_{21}=-J_{1}\left(\Omega_{11}\right) ;
\end{gathered}
$$




$$
\begin{aligned}
& m_{22}=I_{1}\left(\Omega_{11}\right) ; m_{23}=\frac{\lambda_{2}}{\lambda_{1}} J_{1}\left(\Omega_{21}\right) ; m_{24}=\frac{\lambda_{2}}{\lambda_{1}} N_{1}\left(\Omega_{21}\right) \\
& m_{74}=-\left[N_{0}\left(\Omega_{22}\right)-\frac{1-\eta_{2}}{\Omega_{22}} N_{1}\left(\Omega_{22}\right)\right] \\
& \text {; } m_{25}=-\frac{\lambda_{2}}{\lambda_{1}} I_{1}\left(\Omega_{21}\right) ; m_{26}=\frac{\lambda_{2}}{\lambda_{1}} K_{1}\left(\Omega_{21}\right) \text {; } \\
& m_{27} \div m_{2,18}=0 ; P_{2}=0 \text {; } \\
& m_{31}=-\left[J_{0}\left(\Omega_{11}\right)-\frac{1-\eta_{1}}{\Omega_{11}} J_{1}\left(\Omega_{11}\right)\right] \text {; } \\
& m_{32}=\left[I_{0}\left(\Omega_{11}\right)-\frac{1-\eta_{1}}{\Omega_{11}} I_{1}\left(\Omega_{11}\right)\right] \text {; } \\
& m_{33}=\frac{D_{1}^{(2)} \lambda_{2}^{2}}{D_{1}^{(1)} \lambda_{1}^{2}}\left[J_{0}\left(\Omega_{21}\right)-\frac{1-\eta_{2}}{\Omega_{21}} J_{1}\left(\Omega_{21}\right)\right] \text {; } \\
& m_{34}=\frac{D_{1}^{(2)} \lambda_{2}^{2}}{D_{1}^{(1)} \lambda_{1}^{2}}\left[N_{0}\left(\Omega_{21}\right)-\frac{1-\eta_{2}}{\Omega_{21}} N_{1}\left(\Omega_{21}\right)\right] \text {; } \\
& m_{35}=-\frac{D_{1}^{(2)} \lambda_{2}^{2}}{D_{1}^{(1)} \lambda_{1}^{2}}\left[I_{0}\left(\Omega_{21}\right)-\frac{1-\eta_{2}}{\Omega_{21}} I_{1}\left(\Omega_{21}\right)\right] \text {; } \\
& m_{36}=-\frac{D_{1}^{(2)} \lambda_{2}^{2}}{D_{1}^{(1)} \lambda_{1}^{2}}\left[K_{0}\left(\Omega_{21}\right)+\frac{1-\eta_{2}}{\Omega_{21}} K_{1}\left(\Omega_{21}\right)\right] \text {; } \\
& m_{37} \div m_{3,18}=0 ; P_{3}=-\frac{M_{0}^{(2)}}{D_{1}^{(1)} \lambda_{1}^{2}} ; m_{41}=J_{1}\left(\Omega_{11}\right) \text {; } \\
& m_{42}=I_{1}\left(\Omega_{11}\right) ; m_{43}=-\frac{D_{1}^{(2)} \lambda_{2}^{3}}{D_{1}^{(1)} \lambda_{1}^{3}} J_{1}\left(\Omega_{21}\right) ; \\
& m_{44}=-\frac{D_{1}^{(2)} \lambda_{2}^{3}}{D_{1}^{(1)} \lambda_{1}^{3}} N_{1}\left(\Omega_{21}\right) ; m_{45}=-\frac{D_{1}^{(2)} \lambda_{2}^{3}}{D_{1}^{(1)} \lambda_{1}^{3}} I_{1}\left(\Omega_{21}\right) ; \\
& m_{46}=\frac{D_{1}^{(2)} \lambda_{2}^{3}}{D_{1}^{(1)} \lambda_{1}^{3}} K_{1}\left(\Omega_{21}\right) ; m_{47} \div m_{4,18}=0 ; \\
& P_{4}=0 ; m_{51}=m_{52}=0 ; m_{53}=J_{0}\left(\Omega_{22}\right) ; \Omega_{22}=\lambda_{2} R_{2} \text {; } \\
& m_{54}=N_{0}\left(\Omega_{22}\right) ; m_{55}=I_{0}\left(\Omega_{22}\right) ; m_{56}=K_{0}\left(\Omega_{22}\right) \text {; } \\
& m_{57}=-J_{0}\left(\Omega_{12}\right) ; \Omega_{12}=\lambda_{1} R_{2} ; m_{58}=-N_{0}\left(\Omega_{12}\right) ; \\
& m_{59}=-I_{0}\left(\Omega_{12}\right) ; m_{5,10}=-K_{0}\left(\Omega_{12}\right) ; \\
& m_{5,11} \div m_{5,18}=0 ; P_{5}=0 ; m_{61}=m_{62}=0 \text {; } \\
& m_{63}=-J_{1}\left(\Omega_{22}\right) ; m_{64}=-N_{1}\left(\Omega_{22}\right) ; m_{65}=I_{1}\left(\Omega_{22}\right) \text {; } \\
& m_{66}=-K_{1}\left(\Omega_{22}\right) ; m_{67}=\frac{\lambda_{1}}{\lambda_{2}} J_{1}\left(\Omega_{12}\right) ; \\
& m_{68}=\frac{\lambda_{1}}{\lambda_{2}} N_{1}\left(\Omega_{12}\right) ; m_{69}=-\frac{\lambda_{1}}{\lambda_{2}} I_{1}\left(\Omega_{12}\right) ; \\
& m_{6,10}=\frac{\lambda_{1}}{\lambda_{2}} K_{1}\left(\Omega_{12}\right) ; \\
& m_{6,11} \div m_{6,18}=0 ; P_{6}=0 ; m_{71}=m_{72}=0 \text {; } \\
& m_{73}=-\left[J_{0}\left(\Omega_{22}\right)-\frac{1-\eta_{2}}{\Omega_{22}} J_{1}\left(\Omega_{22}\right)\right] \text {; } \\
& m_{75}=\left[I_{0}\left(\Omega_{22}\right)-\frac{1-\eta_{2}}{\Omega_{22}} I_{1}\left(\Omega_{22}\right)\right] \text {; } \\
& m_{76}=\left[K_{0}\left(\Omega_{22}\right)+\frac{1-\eta_{2}}{\Omega_{22}} K_{1}\left(\Omega_{22}\right)\right] \text {; } \\
& m_{77}=\frac{D_{1}^{(1)} \lambda_{1}^{2}}{D_{1}^{(2)} \lambda_{2}^{2}}\left[J_{0}\left(\Omega_{12}\right)-\frac{1-\eta_{1}}{\Omega_{12}} J_{1}\left(\Omega_{12}\right)\right] \text {; } \\
& m_{78}=\frac{D_{1}^{(1)} \lambda_{1}^{2}}{D_{1}^{(2)} \lambda_{2}^{2}}\left[N_{0}\left(\Omega_{12}\right)-\frac{1-\eta_{1}}{\Omega_{12}} N_{1}\left(\Omega_{12}\right)\right] \text {; } \\
& m_{79}=-\frac{D_{1}^{(1)} \lambda_{1}^{2}}{D_{1}^{(2)} \lambda_{2}^{2}}\left[I_{0}\left(\Omega_{12}\right)-\frac{1-\eta_{1}}{\Omega_{12}} I_{1}\left(\Omega_{12}\right)\right] \text {; } \\
& m_{7,10}=-\frac{D_{1}^{(1)} \lambda_{1}^{2}}{D_{1}^{(2)} \lambda_{2}^{2}}\left[K_{0}\left(\Omega_{12}\right)+\frac{1-\eta_{1}}{\Omega_{12}} K_{1}\left(\Omega_{12}\right)\right] \text {; } \\
& m_{7,11} \div m_{7,18}=0 ; P_{7}=\frac{M_{0}^{(2)}}{D_{1}^{(2)} \lambda_{2}^{2}} ; m_{81} \div m_{82}=0 \text {; } \\
& m_{83}=J_{1}\left(\Omega_{22}\right) ; m_{84}=N_{1}\left(\Omega_{22}\right) ; m_{85}=I_{1}\left(\Omega_{22}\right) \text {; } \\
& m_{86}=-K_{1}\left(\Omega_{22}\right) ; m_{87}=-\frac{D_{1}^{(1)} \lambda_{1}^{3}}{D_{1}^{(2)} \lambda_{2}^{3}} J_{1}\left(\Omega_{12}\right) ; \\
& m_{88}=-\frac{D_{1}^{(1)} \lambda_{1}^{3}}{D_{1}^{(2)} \lambda_{2}^{3}} N_{1}\left(\Omega_{12}\right) ; m_{89}=-\frac{D_{1}^{(1)} \lambda_{1}^{3}}{D_{1}^{(2)} \lambda_{2}^{3}} I_{1}\left(\Omega_{12}\right) \text {; } \\
& m_{8,10}=\frac{D_{1}^{(1)} \lambda_{1}^{3}}{D_{1}^{(2)} \lambda_{2}^{3}} K_{1}\left(\Omega_{12}\right) ; m_{8,11} \div m_{8,18}=0 ; \\
& P_{8}=0 ; m_{91} \div m_{96}=0 ; m_{97}=J_{0}\left(\Omega_{13}\right) ; \Omega_{13}=\lambda_{1} R_{3} \text {; } \\
& m_{98}=N_{0}\left(\Omega_{13}\right) ; m_{99}=I_{0}\left(\Omega_{13}\right) ; m_{9,10}=K_{0}\left(\Omega_{13}\right) ; \\
& m_{9,11}=-J_{0}\left(\Omega_{23}\right) ; \Omega_{23}=\lambda_{2} R_{3} ; m_{9,12}=-N_{0}\left(\Omega_{23}\right) \text {; } \\
& m_{9,13}=-I_{0}\left(\Omega_{23}\right) ; m_{9,14}=-K_{0}\left(\Omega_{23}\right) ; \\
& m_{9,15} \div m_{9,18}=0 ; P_{9}=0 ; m_{10,1} \div m_{10,6}=0 \text {; } \\
& m_{10,7}=-J_{1}\left(\Omega_{13}\right) ; m_{10,8}=-N_{1}\left(\Omega_{13}\right) ; m_{10,9}=I_{1}\left(\Omega_{13}\right) \text {; } \\
& m_{10,10}=-K_{1}\left(\Omega_{13}\right) ; m_{10,11}=\frac{\lambda_{2}}{\lambda_{1}} J_{1}\left(\Omega_{23}\right) \text {; } \\
& m_{10,12}=\frac{\lambda_{2}}{\lambda_{1}} N_{1}\left(\Omega_{23}\right) ; m_{10,13}=-\frac{\lambda_{2}}{\lambda_{1}} I_{1}\left(\Omega_{23}\right) ; \\
& m_{10,14}=\frac{\lambda_{2}}{\lambda_{1}} K_{1}\left(\Omega_{23}\right) ; m_{10,15} \div m_{10,18}=0 ; P_{10}=0 \text {; } \\
& m_{11,1} \div m_{11,6}=0 \text {; } \\
& m_{11,7}=-\left[J_{0}\left(\Omega_{13}\right)-\frac{1-\eta_{1}}{\Omega_{13}} J_{1}\left(\Omega_{13}\right)\right] \text {; } \\
& m_{11,8}=-\left[N_{0}\left(\Omega_{13}\right)-\frac{1-\eta_{1}}{\Omega_{13}} N_{1}\left(\Omega_{13}\right)\right] \text {; }
\end{aligned}
$$




$$
\begin{aligned}
& m_{11,9}=\left[I_{0}\left(\Omega_{13}\right)-\frac{1-\eta_{1}}{\Omega_{13}} I_{1}\left(\Omega_{13}\right)\right] ; \\
& m_{11,10}=\left[K_{0}\left(\Omega_{13}\right)+\frac{1-\eta_{1}}{\Omega_{13}} K_{1}\left(\Omega_{13}\right)\right] \text {; } \\
& m_{11,11}=\frac{D_{1}^{(2)} \lambda_{2}^{2}}{D_{1}^{(1)} \lambda_{1}^{2}}\left[J_{0}\left(\Omega_{23}\right)-\frac{1-\eta_{2}}{\Omega_{23}} J_{1}\left(\Omega_{23}\right)\right] ; \\
& m_{11,12}=\frac{D_{1}^{(2)} \lambda_{2}^{2}}{D_{1}^{(1)} \lambda_{1}^{2}}\left[N_{0}\left(\Omega_{23}\right)-\frac{1-\eta_{2}}{\Omega_{23}} N_{1}\left(\Omega_{23}\right)\right] \text {; } \\
& m_{11,13}=-\frac{D_{1}^{(2)} \lambda_{2}^{2}}{D_{1}^{(1)} \lambda_{1}^{2}}\left[I_{0}\left(\Omega_{23}\right)-\frac{1-\eta_{2}}{\Omega_{23}} I_{1}\left(\Omega_{23}\right)\right] \text {; } \\
& m_{11,14}=-\frac{D_{1}^{(2)} \lambda_{2}^{2}}{D_{1}^{(1)} \lambda_{1}^{2}}\left[K_{0}\left(\Omega_{23}\right)+\frac{1-\eta_{2}}{\Omega_{23}} K_{1}\left(\Omega_{23}\right)\right] ; \\
& m_{11,15} \div m_{11,18}=0 ; P_{11}=-\frac{M_{0}^{(4)}}{D_{1}^{(1)} \lambda_{1}^{2}} ; m_{12,1} \div m_{12,6}=0 \text {; } \\
& m_{12,7}=J_{1}\left(\Omega_{13}\right) ; m_{12,8}=N_{1}\left(\Omega_{13}\right) ; \\
& m_{12,9}=I_{1}\left(\Omega_{13}\right) ; m_{12,10}=-K_{1}\left(\Omega_{13}\right) \text {; } \\
& m_{12,11}=-\frac{D_{1}^{(2)} \lambda_{2}^{3}}{D_{1}^{(1)} \lambda_{1}^{3}} J_{1}\left(\Omega_{23}\right) ; m_{12,12}=-\frac{D_{1}^{(2)} \lambda_{2}^{3}}{D_{1}^{(1)} \lambda_{1}^{3}} N_{1}\left(\Omega_{23}\right) \text {; } \\
& m_{12,13}=-\frac{D_{1}^{(2)} \lambda_{2}^{3}}{D_{1}^{(1)} \lambda_{1}^{3}} I_{1}\left(\Omega_{23}\right) ; m_{12,14}=\frac{D_{1}^{(2)} \lambda_{2}^{3}}{D_{1}^{(1)} \lambda_{1}^{3}} K_{1}\left(\Omega_{23}\right) ; \\
& m_{12,15} \div m_{12,18}=0 ; P_{12}=0 \text {; } \\
& m_{13,1} \div m_{13,10}=0 ; m_{13,11}=J_{0}\left(\Omega_{24}\right) ; \Omega_{24}=\lambda_{2} R_{4} \text {; } \\
& m_{13,12}=N_{0}\left(\Omega_{24}\right) ; m_{13,13}=I_{0}\left(\Omega_{24}\right) ; \\
& m_{13,14}=K_{0}\left(\Omega_{24}\right) ; m_{13,15}=-J_{0}\left(\Omega_{4}\right) ; \Omega_{4}=\lambda R_{4} ; \\
& m_{13,16}=-N_{0}\left(\Omega_{4}\right) ; m_{13,17}=-I_{0}\left(\Omega_{4}\right) ; \\
& m_{13,18}=-K_{0}\left(\Omega_{4}\right) ; P_{13}=0 ; m_{14,1} \div m_{14,10}=0 \text {; } \\
& m_{14,11}=-J_{1}\left(\Omega_{24}\right) ; m_{14,12}=-N_{1}\left(\Omega_{24}\right) ; \\
& m_{14,13}=I_{1}\left(\Omega_{24}\right) ; m_{14,14}=-K_{1}\left(\Omega_{24}\right) \text {; } \\
& m_{14,15}=\frac{\lambda}{\lambda_{2}} J_{1}\left(\Omega_{4}\right) ; m_{14,16}=\frac{\lambda}{\lambda_{2}} N_{1}\left(\Omega_{4}\right) \text {; } \\
& m_{14,17}=-\frac{\lambda}{\lambda_{2}} I_{1}\left(\Omega_{4}\right) ; \\
& m_{14,18}=\frac{\lambda}{\lambda_{2}} K_{1}\left(\Omega_{4}\right) ; P_{14}=0 ; m_{15,1} \div m_{15,10}=0 \text {; } \\
& m_{15,11}=-\left[J_{0}\left(\Omega_{24}\right)-\frac{1-\eta_{2}}{\Omega_{24}} J_{1}\left(\Omega_{24}\right)\right] ; \\
& m_{15,12}=-\left[N_{0}\left(\Omega_{24}\right)-\frac{1-\eta_{2}}{\Omega_{24}} N_{1}\left(\Omega_{24}\right)\right] \text {; } \\
& m_{15,13}=\left[I_{0}\left(\Omega_{24}\right)-\frac{1-\eta_{2}}{\Omega_{24}} I_{1}\left(\Omega_{24}\right)\right] ;
\end{aligned}
$$

(C) О. М. Петрищев, К. В. Базіло, 2019 DOI: $10.24025 / 2306-4412.3 .2019 .173067$

$$
\begin{gathered}
m_{15,14}=\left[K_{0}\left(\Omega_{24}\right)+\frac{1-\eta_{2}}{\Omega_{24}} K_{1}\left(\Omega_{24}\right)\right] ; \\
m_{15,15}=\frac{D_{u} \lambda^{2}}{D_{1}^{(2)} \lambda_{2}^{2}}\left[J_{0}\left(\Omega_{4}\right)-\frac{1-v}{\Omega_{4}} J_{1}\left(\Omega_{4}\right)\right] ; \\
m_{15,16}=\frac{D_{\mu} \lambda^{2}}{D_{1}^{(2)} \lambda_{2}^{2}}\left[N_{0}\left(\Omega_{4}\right)-\frac{1-v}{\Omega_{4}} N_{1}\left(\Omega_{4}\right)\right] ; \\
m_{15,17}=-\frac{D_{\mu} \lambda^{2}}{D_{1}^{(2)} \lambda_{2}^{2}}\left[I_{0}\left(\Omega_{4}\right)-\frac{1-v}{\Omega_{4}} I_{1}\left(\Omega_{4}\right)\right] ; \\
m_{15,18}=-\frac{D_{u} \lambda^{2}}{D_{1}^{(2)} \lambda_{2}^{2}}\left[K_{0}\left(\Omega_{4}\right)+\frac{1-v}{\Omega_{4}} K_{1}\left(\Omega_{4}\right)\right] ; \\
P_{15}=\frac{M_{0}^{(4)}}{D_{1}^{(2)} \lambda_{2}^{2}} ; m_{16,1} \div m_{16,10}=0 ; m_{16,11}=J_{1}\left(\Omega_{24}\right) ; \\
m_{16,12}=N_{1}\left(\Omega_{24}\right) ; m_{16,13}=I_{1}\left(\Omega_{24}\right) ; \\
m_{16,14}=-K_{1}\left(\Omega_{24}\right) ; m_{16,15}=-\frac{D_{\mu} \lambda^{3}}{D_{1}^{(2)} \lambda_{2}^{3}} J_{1}\left(\Omega_{4}\right) ; \\
m_{16,16}=-\frac{D_{\mu} \lambda^{3}}{D_{1}^{(2)} \lambda_{2}^{3}} N_{1}\left(\Omega_{4}\right) ; m_{16,17}=-\frac{D_{\mu} \lambda^{3}}{D_{1}^{(2)} \lambda_{2}^{3}} I_{1}\left(\Omega_{4}\right) ; \\
m_{16,18}=\frac{D_{u} \lambda^{3}}{D_{1}^{(2)} \lambda_{2}^{3}} K_{1}\left(\Omega_{4}\right) ; P_{16}=0 ; \\
m_{17,1} \div m_{17,14}=0 ; m_{17,15}=J_{0}(\Omega) ; \Omega=\lambda R ; \\
m_{17,16}=N_{0}(\Omega) ; m_{17,17}=I_{0}(\Omega) ; m_{17,18}=K_{0}(\Omega) ; \\
P_{17}=0 ; m_{18,1} \div m_{18,14}=0 ; m_{18,15}=-J_{1}(\Omega) ; \\
m_{18,16}=-N_{1}(\Omega) ; m_{18,17}=I_{1}(\Omega) ; \\
m_{18,18}=-K_{1}(\Omega) ; P_{18}=0 .
\end{gathered}
$$

У разі, коли $U_{2}^{(-)}=U_{2}^{(+)}=U_{2}$, загальне рішення системи рівнянь (13) записується в наступному вигляді:

$$
A_{k}=(-1)^{k}\left(\beta_{k 2} U_{2}+\beta_{k 0} U_{0}\right),
$$

де $\beta_{k 2}$ i $\beta_{k 0}$ - чутливості зміщень матеріальних частинок (розмірність метр, поділений на вольт) біморфного п'єзоелектричного трансформатора до потенціалів $U_{2}$ і $U_{0}$. Числові значення чутливості $\beta_{k 2}$ і $\beta_{k 0}$ задаються наступними виразами:

$$
\begin{aligned}
& \beta_{k 2}=\frac{2 e_{31}^{*}(h+\alpha / 2)}{D_{1}^{(1)} \lambda_{1}^{2} \Delta_{0}}\left[\Delta_{k 3}-\frac{D_{1}^{(1)} \lambda_{1}^{2}}{D_{1}^{(2)} \lambda_{2}^{2}} \Delta_{k 7}\right], \\
& \beta_{k 0}=\frac{2 e_{31}^{*}(h+\alpha / 2)}{D_{1}^{(1)} \lambda_{1}^{2} \Delta_{0}}\left[\Delta_{k, 11}-\frac{D_{1}^{(1)} \lambda_{1}^{2}}{D_{1}^{(2)} \lambda_{2}^{2}} \Delta_{k, 15}\right],
\end{aligned}
$$

де $\Delta_{0}$ - головний визначник системи рівнянь (13), тобто визначник матриці розміром $18 \times 18$, складеної 3 коефіцієнтів при шуканих константах $A_{1}, \ldots, A_{18} ; \Delta_{k j}(j=3 ; 7 ; 11 ; 15)$ - ви- 
значники матриць розміром $17 \times 17$, які виходять 3 вихідної матриці викреслюванням $k$-го стовпця i $j$-го рядка.

Для того щоб знайти коефіцієнт електроакустичного зв'язку між потенціалами $U_{2} \mathrm{i}$ $U_{0}$, визначимо потенціал $U_{2}$.

Так як $U_{2}^{(-)}=U_{2}^{(+)}=U_{2}$, то досить визначити один з струмів $I_{2}^{( \pm)}$у провіднику, який з'єднує один із електродів вторинного електричного кола п'єзоелектричного трансформатора 3 відповідним електричним навантаженням. Амплітудне значення $I_{2}^{(+)}$струму в навантаженні $Z_{2}^{(+)}$визначається стандартним чином

$$
I_{2}^{(+)}=-i \omega Q_{2}^{(+)}=-2 \pi i \omega \int_{R_{1}}^{R_{2}} \rho^{(2)} D_{z}^{(+)}(\rho) d \rho .
$$

Підставляючи в формулу (15) визначення ${ }^{(2)} D_{z}^{(+)}(\rho)$, задане виразом (4), отримуємо наступний результат:

$$
I_{2}^{(+)}=2 \pi i \omega e_{31}^{*}(h+\alpha / 2)\left[\left.R_{2} \frac{\partial w_{2}(\rho)}{\partial \rho}\right|_{\rho=R_{2}}-\left.R_{1} \frac{\partial w_{2}(\rho)}{\partial \rho}\right|_{\rho=R_{1}}\right]+
$$$$
+i \omega C_{2}^{\sigma} U_{2}^{(+)},
$$

де $C_{2}^{\sigma}=\pi\left(R_{2}^{2}-R_{1}^{2}\right) \chi_{33}^{\sigma} / \alpha$ - динамічна електрична ємність кільцевої області верхнього п'єзокерамічного диска під електродом вторинного електричного кола п'єзоелектричного трансформатора.

Першу похідну функції $w_{2}(\rho)$, яка пропорційна кутам поворотів циліндричних перерізів кільцевої області $R_{1} \leq \rho \leq R_{2}$ всього п'єзоелектричного трансформатора, 3 урахуванням виразу (14) можна записати в наступному вигляді:

$$
\begin{gathered}
\rho \frac{\partial w_{2}(\rho)}{\partial \rho}=\lambda_{2} \rho\left[\Xi_{22}\left(\lambda_{2} \rho\right) U_{2}^{(+)}+\Xi_{20}\left(\lambda_{2} \rho\right) U_{0}\right], \\
\Xi_{2 j}\left(\lambda_{2} \rho\right)=\beta_{3 j} J_{1}\left(\lambda_{2} \rho\right)-\beta_{4 j} N_{1}\left(\lambda_{2} \rho\right)+ \\
+\beta_{5 j} I_{1}\left(\lambda_{2} \rho\right)-\beta_{6 j} K_{1}\left(\lambda_{2} \rho\right)
\end{gathered}
$$

де $(j=0 ; 2)$; частотно залежні коефіцієнти $\beta_{k j}$ визначені в коментарях до формули (14).

Використовуючи розрахункове співвідношення (17) у формулі (16), доходимо висновку, що

$I_{2}^{(+)}=2 \pi i \omega e_{31}^{*}(h+\alpha / 2)\left[\Psi_{22}\left(\lambda_{2}, \Gamma_{2}\right) U_{2}^{(+)}+\Psi_{22}\left(\lambda_{2}, \Gamma_{2}\right) U_{0}\right]+$ $+i \omega C_{2}^{\sigma} U_{2}^{(+)}$, де $\Psi_{2 j}\left(\lambda_{2}, \Gamma_{2}\right)=\lambda_{2} R_{2} \Xi_{2 j}\left(\lambda_{2} R_{2}\right)-\lambda_{2} R_{1} \Xi_{2 j}\left(\lambda_{2} R_{1}\right)$, $(j=0 ; 2)$; символ $\Gamma_{2}$ означає набір геометричних параметрів області № 2.

Оскільки $U_{2}^{(+)}=Z_{2}^{(+)} I_{2}^{(+)}$, то 3 визначення (18) випливає, що $U_{2}^{(+)}\left[1-i \omega C_{2}^{\sigma} Z_{2}^{(+)}-2 \pi i \omega Z_{2}^{(+)} e_{31}^{*}(h+\alpha / 2) \Psi_{22}\left(\lambda_{2}, \Gamma_{2}\right)\right]=$ $=2 \pi i \omega Z_{2}^{(+)} e_{31}^{*}(h+\alpha / 2) \Psi_{20}\left(\lambda_{2}, \Gamma_{2}\right) U_{0}$.

3 останньої рівності визначається коефіцієнт електроакустичного зв'язку $K_{0}^{(2)}\left(\omega, \Pi_{2}\right)$ :

$$
\begin{aligned}
& K_{0}^{(2)}\left(\omega, \Pi_{2}\right)=\frac{U_{2}^{(+)}}{U_{0}}= \\
& =\frac{2 \pi i \omega Z_{2}^{(+)} e_{31}^{*}(h+\alpha / 2) \Psi_{20}\left(\lambda_{2}, \Gamma_{2}\right)}{1-i \omega C_{2}^{\sigma} Z_{2}^{(+)}-2 \pi i \omega Z_{2}^{(+)} e_{31}^{*}(h+\alpha / 2) \Psi_{22}\left(\lambda_{2}, \Gamma_{2}\right)},
\end{aligned}
$$

де символом $\Pi_{2}$ позначений набір геометричних, фізико-механічних та електричних параметрів області № 2 біморфного п'єзоелектричного трансформатора.

Для завершення побудови математичної моделі дискового біморфного п'єзоелектричного трансформатора необхідно визначити потенціал $U_{0}$ на кільцевих електродах первинного електричного кола.

Дотримуючись того, що було отримано раніше, можна записати, що

$$
U_{0}=\frac{U_{1} Z_{L}^{(4)}}{Z_{5}^{(4)}+Z_{1}}=\frac{U_{1} Z_{4}^{(+)}}{Z_{4}^{(+)}+2 Z_{1}},
$$

де $Z_{b}^{(4)}=Z_{4}^{(+)} / 2$ - електричний імпеданс області № 4 біморфного п'єзоелектричного трансформатора; $Z_{4}^{(+)}$- електричний імпеданс кільцевої області № 4 верхнього п'єзокерамічного диска. Електричний імпеданс $Z_{4}^{(+)}$визначається законом Ома для ділянки електричного кола, який записується в такий спосіб

$$
Z_{4}^{(+)}=\frac{U_{0}}{I_{4}^{(+)}}=\frac{U_{0}}{-i \omega Q_{4}^{(+)}},
$$

де $I_{4}^{(+)}$i $Q_{4}^{(+)}$- амплітудне значення струму в провіднику і електричний заряд на кільцевому електроді первинного електричного кола.

3 визначення (4) випливає

$Q_{4}^{(+)}=-2 \pi e_{31}^{*}(h+\alpha / 2)\left[\left.R_{4} \frac{\partial w_{4}(\rho)}{\partial \rho}\right|_{\rho=R_{4}}-\left.R_{3} \frac{\partial w_{4}(\rho)}{\partial \rho}\right|_{\rho=R_{3}}\right]-$

$-C_{4}^{\sigma} U_{0}$

де $C_{4}^{\sigma}=\pi\left(R_{4}^{2}-R_{3}^{2}\right) \chi_{33}^{\sigma} / \alpha-$ динамічна електрична ємність кільцевого об'єму верхнього п'єзокерамічного диска п'єзоелектричного трансформатора. 
Оскільки $\rho \frac{\partial w_{4}(\rho)}{\partial \rho}=U_{0} \Xi_{40}\left(\lambda_{2} \rho\right)$,

де

$$
\begin{aligned}
\Xi_{40}\left(\lambda_{2} \rho\right) & =\lambda_{2} \rho\left\{\left[\beta_{11,2} K_{0}^{(2)}\left(\omega, \Pi_{2}\right)+\beta_{11,0}\right] J_{1}\left(\lambda_{2} \rho\right)-\right. \\
- & {\left[\beta_{12,2} K_{0}^{(2)}\left(\omega, \Pi_{2}\right)+\beta_{12,0}\right] N_{1}\left(\lambda_{2} \rho\right)-} \\
- & {\left[\beta_{13,2} K_{0}^{(2)}\left(\omega, \Pi_{2}\right)+\beta_{13,0}\right] I_{1}\left(\lambda_{2} \rho\right)-} \\
- & {\left.\left[\beta_{14,2} K_{0}^{(2)}\left(\omega, \Pi_{2}\right)+\beta_{14,0}\right] K_{1}\left(\lambda_{2} \rho\right)\right\}, }
\end{aligned}
$$

то вираз (22) можна записати в такому вигляді:

$Q_{4}^{(+)}=-C_{4}^{\sigma} U_{0}\left[\Psi_{40}\left(\lambda_{2}, \Gamma_{4}\right)+1\right]$,

де

$\Psi_{40}\left(\lambda_{2}, \Gamma_{4}\right)=\frac{2 e_{31}^{*}(h+\alpha / 2) \alpha}{\left(R_{4}^{2}-R_{3}^{2}\right) \chi_{33}^{\sigma}}\left[\Xi_{40}\left(\lambda_{2} R_{4}\right)-\Xi_{40}\left(\lambda_{2} R_{3}\right)\right] ;$

символ $\Gamma_{4}$ означає набір геометричних параметрів області № 4.

Підставляючи вираз (23) у визначення

(21) електричного імпедансу $Z_{4}^{(+)}$, отримуємо наступний результат:

$$
Z_{4}^{(+)}=\frac{1}{i \omega C_{4}^{\sigma}\left[\Psi_{40}\left(\lambda_{2}, \Gamma_{4}\right)+1\right]} .
$$

Після цього 3 виразу (20) визначається потенціал $U_{0}$ :

$$
U_{0}=\frac{U_{1}}{1+2 i \omega C_{4}^{\sigma} Z_{1}\left[\Psi_{40}\left(\lambda_{2}, \Gamma_{4}\right)+1\right]} .
$$

Підставляючи знайдене значення $U_{0} \mathrm{y}$ формулу (19), знаходимо, що

$$
\frac{U_{2}^{(+)}}{U_{0}}=\frac{U_{2}^{(+)}}{U_{1}}\left\{1+2 i \omega C_{4}^{\sigma} Z_{1}\left[\Psi_{40}\left(\lambda_{2}, \Gamma_{4}\right)+1\right]\right\}=K_{0}^{(2)}\left(\omega, \Pi_{2}\right) .
$$

3 останнього запису випливає, що

$$
K^{(+)}(\omega, \Pi)=\frac{K_{0}^{(2)}\left(\omega, \Pi_{2}\right)}{1+2 i \omega C_{4}^{\sigma} Z_{1}\left[\Psi_{40}\left(\lambda_{2}, \Gamma_{4}\right)+1\right]} .
$$

Вираз (24) має сенс математичної моделі дискового біморфного п'єзоелектричного трансформатора 3 симетричним електричним навантаженням у вторинному електричному колі.

Висновки. Основний результат статті можна зафіксувати наступним чином: розглянуто конструкцію і особливості математичного опису біморфного п'єзоелектричного елемента, принцип дії якого базується на використанні осесиметричних коливань поперечного вигину; отримано рішення задачі про порушення коливань поперечного вигину в біморфному п'єзоелектричному елементі. Побудовано математичну модель дискового біморфного п'єзоелектричного трансформатора 3 симетрич-

(C) О. М. Петрищев, К. В. Базіло, 2019 DOI: $10.24025 / 2306-4412.3 .2019 .173067$ ним електричним навантаженням у вторинному електричному колі з урахуванням повного набору геометричних, фізико-механічних та електричних параметрів.

\section{Список літератури}

[1] С. Жуков, "Пьезокерамика и перспективы ее применения", MTT. Tехно plus, № 5, с. 56-60, 2009.

[2] Ю. А. Смирнов, и А. В. Муханов, Электронные и микропрочессорные системы управления автомобилей: учеб. пособ. Санкт-Петербург: Лань, 2012.

[3] T. Bove, W. Wolny, E. Ringgaard, and K. Breboel, "New type of piezoelectric transformer with very high power density", Applications of Ferroelectrics, vol. 1, pp. 321-324, 2000. doi: 10.1109/ISAF. 2000.941564.

[4] A. M. Flynn, and S. R. Sanders, "Fundamental limits on energy transfer and circuit considerations for piezoelectric transformers", IEEE Transactions on Power Electronics, vol. 17, pp. 8-14, 2002. doi: $10.1109 / 63.988662$.

[5] W. Shao, L. Chen, C. Pan, Y. Liu, and Z. Feng, "Power density of piezoelectric transformers improved using a contact heat transfer structure", IEEE Transactions on Ultrasonics, Ferroelectrics and Frequency Control, vol. 59, pp. 73-81, 2012. doi: 10.1109/TUFFC.2012.2157.

[6] E. Horsley, M. Foster, and D. Stone, "Stateof-the-art piezoelectric transformer technology", in EPE, 2007, pp. 1-10.

[7] T. Andersen, M. A. E. Andersen, and O. C. Thomsen, "Simulation of piezoelectric transformers with COMSOL", in Proc. 2012 COMSOL Conf. in Milan. [Online]. Available:https://www.comsol.dk/paper/download/ 151765/andersen_paper.pdf

[8] А. А. Ерофеев, Г. А. Данов, и В. Н. Фролов, Пьезокерамические трансформаторы и их применение в радиоэлектронике. Москва: Радио и связь, 1988.

[9] А. И. Ладик, и А. И. Сташкевич, Изделия электронной техники. Пьезоэлектрические и электромеханические приборы. Москва: Радио и связь, 1993.

[10] С. Г. Бочкарев, Б. Б. Педько, и В. П. Каменцев, "Амплитудный и частотный способы управления работой пьезотрансформаторов в радиоэлектронных устрой- 
ствах", Вестник ТвГУ. Серия: Физика, № 4 (6), c. 110-114, 2004.

[11] В. В. Лавриненко, Пьезоэлектрические трансформаторы. Москва: Энергия, 1975.

[12] А. В. Богдан, О. Н. Петрищев, Ю. И. Якименко, и Ю. Ю. Яновская, "Исследование характеристик пьезоэлектрического трансформатора на основе радиальных колебаний в тонких пьезокерамических дисках", Электроника и связь. Тематический выпуск: Электроника и нанотехнологии, ч. 1, с. 269-274, 2009.

[13] А. В. Богдан, О. Н. Петрищев, Ю. И. Якименко, и Ю. Ю. Яновская, "Математическое моделирование колебаний тонких пьезокерамических дисков для создания функциональных элементов пьезоэлектроники", Электроника и связь. Тематический выпуск: Электроника и нанотехнологии, ч. 2, с. 35-42, 2009.

[14] M. Peerasaksophol, S. Srilomsak, P. Laoratanakul, and T. Kulworawanichpong, "Design and implementation of ring-dot piezoelectric ballasts for 36-W fluorescent lamps", European Journal of Scientific Research, vol. 64, no. 2, pp. 189-205, 2011.

[15] D. Livingston, K. P. Kumar, and N. Venugopal, "Modelling and simulation of multiple piezo-electric transformer converters", International Journal of Emerging Technology and Advanced Engineering, vol. 3, no. 8, pp. 237-245, 2013.

[16] О.Н.Петрищев, и К.В.Базило, "Принципы математического моделирования трансформаторов, работающих на планарных осесимметричных колебаниях пьезокерамических дисков", Вісник Черкаського державного технологічного університету, № 3, с. 10-20, 2015 (Технічні науки).

\section{References}

[1] S. Zhukov, "Piezoceramics and prospects of its application", MTT. Tekhno plus, no. 5, pp. 56-60, 2009 [in Russian].

[2] Yu. A. Smirnov, and A. V. Mukhanov, Electronic and microprocessor control systems of cars. St. Petersburg, Lan, 2012 [in Russian].

[3] T. Bove, W. Wolny, E. Ringgaard, and K. Breboel, "New type of piezoelectric transformer with very high power density",
Applications of Ferroelectrics, vol. 1, pp. 321-324, 2000.

doi: 10.1109/ISAF.2000.941564.

[4] A. M. Flynn, and S. R. Sanders, "Fundamental limits on energy transfer and circuit considerations for piezoelectric transformers", IEEE Transactions on Power Electronics, vol. 17, pp. 8-14, 2002.

doi: 10.1109/63.988662.

[5] W. Shao, L. Chen, C. Pan, Y. Liu, and Z. Feng, "Power density of piezoelectric transformers improved using a contact heat transfer structure", IEEE Transactions on Ultrasonics, Ferroelectrics and Frequency Control, vol. 59, pp. 73-81, 2012.

doi: 10.1109/TUFFC.2012.2157.

[6] E. Horsley, M. Foster, and D. Stone, "Stateof-the-art piezoelectric transformer technology", in EPE, pp. 1-10, 2007.

[7] T. Andersen, M. A. E. Andersen, and O. C. Thomsen, "Simulation of piezoelectric transformers with COMSOL", in Proc. 2012 COMSOL Conf. in Milan. [Online]. Available:https://www.comsol.dk/paper/download/ 151765/andersen_paper.pdf

[8] A. A. Erofeev, G. A. Danov, and V. N. Frolov, Piezoceramic transformers and their application in radio electronics. Moscow, Radio i svyaz, 1988 [in Russian].

[9] A. I. Ladik, and A. I. Stashkevich, Electronic products. Piezoelectric and electromechanical devices. Moscow, Radio i svyaz, 1993 [in Russian].

[10] S. G. Bochkarev, B. B. Pedko, and V. P. Kamentsev, "Amplitude and frequency methods of controlling the operation of piezotransformers in electronic devices", Vestnik TvGU. Seriya: Fizika, no. 4 (6), pp. 110114, 2004 [in Russian].

[11] V. V. Lavrinenko, Piezoelectric transformers. Moscow: Energiya, 1975 [in Russian].

[12] A. V. Bogdan, O. N. Petrischev, Yu. I. Yakimenko, and Yu. Yu. Yanovskaya, "Investigation of characteristics of piezoelectric transformer based on radial oscillations in thin piezoceramic disks", Elektronika $i$ svyaz. Tematicheskiy vyipusk: Elektronika i nanotehnologii, part 1, pp. 269-274, 2009 [in Russian].

[13] A. V. Bogdan, O. N. Petrischev, Yu. I. Yakimenko, and Yu. Yu. Yanovskaya, "Mathematical modelling of oscillations of thin 
piezoceramic disks for creating functional elements of piezoelectronics", Elektronika i svyaz. Tematicheskiy vyipusk: Elektronika i nanotehnologii, part 2, pp. 35-42, 2009 [in Russian].

[14] M. Peerasaksophol, S. Srilomsak, P. Laoratanakul, and T. Kulworawanichpong, "Design and implementation of ring-dot piezoelectric ballasts for 36-W fluorescent lamps", European Journal of Scientific Research, vol. 64, no. 2, pp. 189-205, 2011.
[15] D. Livingston, K. P. Kumar, and N. Venugopal, "Modelling and simulation of multiple piezoelectric transformer converters", International Journal of Emerging Technology and Advanced Engineering, vol. 3, no. 8, pp. 237-245, 2013.

[16] O. N. Petrishchev, and C. V. Bazilo, "Principles of mathematical modeling of transformers operating on planar axisymmetric oscillations of piezoceramic discs", Visnyk Cherkaskogo derzhavnogo tehnologichnogo universitetu, no. 3, pp. 10-20, 2015 [in Russian].

O. N. Petrishchev, Dr. Sc. (Eng.), professor, Kyiv Scientific Research Institute of Hydrodevices

C. V. Bazilo, Ph. D. (Eng.), associate professor Cherkasy State Technological University Shevchenko blvd, 460, Cherkassy, 18006, Ukraine

\section{CONSTRUCTION OF MATHEMATICAL MODEL OF BIMORPH PIEZOELECTRIC TRANSFORMER}

Introduction. Piezoceramic transformers are most widely used in high-voltage sources of secondary power supply and in devices where a large voltage conversion ratio is needed, in particular, in medical technology.

Purpose is to solve the problem of the excitation of transverse bending oscillations in bimorph piezoelectric transformer. The ultimate goal of mathematical modelling of physical state of oscillating piezoceramic elements consists in qualitative and quantitative description of characteristics and parameters of electric and elastic fields existing in them.

Methodology. Mathematical model of piezoelectric transformers operation using transverse bending vibrations in a bimorph piezoelectric element is constructed with a minimal number of assumptions simplifying the real situation. This allows to state that the proposed construction scheme delivers mathematical models that are sufficiently adequate to real objects and physical processes that exist in them.

Results. Construction and features of mathematical description of bimorph piezoelectric element, the principle of which is based on the use of axisymmetric transverse bending oscillations, are considered. The solution of the problem of transverse bending oscillations excitation in bimorph piezoelectric element by the difference of electric potentials is obtained.

Originality. Mathematical model of disk bimorph piezoelectric transformer with symmetrical electrical loads in secondary electrical circuit taking into account a complete set of geometrical, physical, mechanical and electrical parameters is built.

Practical value. As a result of research of real device's mathematical model a set of geometrical, physical, mechanical and electrical parameters of a real object can be determined which provides the realization of technical parameters of piezoelectric functional element specified in technical specifications. The cost of the saved resources is the commercial price of mathematical model.

Keywords: piezoelectric transformer, bimorph element, physical processes, mathematical description.

Стаття надійшла 10.07.2019

Прийнято 12.08.2019 